\title{
AUTOMATION SYSTEM IN LABVIEW FOR PARAMETERIZATION AND UNSUPERVISED DATA ACQUISITION IN A HIGH-TEMPERATURE OVEN
}

\author{
WILMAR A. MONTAÑO R., JULIÁN R. CAMARGO L. \& CÉSAR A. PERDOMO CH.
}

Engineering Faculty, Universidad Distrital Francisco José de Caldas, Bogotá, Colombia

\section{ABSTRACT}

This paper presents a system for automating the parameterization and unsupervised data collection from a high-temperature furnace that is used as a comparison standard in the calibration of platinum resistance thermometers. For this entire process, the LabView software is used, with which an application is developed that allows the configuration of the desired operating temperature and automatic data acquisition.

KEYWORDS: CRC, LabView, Metrology, Modbus, Temperature

Received: Dec 02, 2020; Accepted: Dec 22, 2020; Published: Dec 16, 2020; Paper Id.: IJMPERDDEC202031

\section{INTRODUCTION}

Accurate temperature measurement is vital in a wide range of human activities, it is one of the key parameters to measure in industry, meteorology, health care and science among others [1]. This magnitude is related to the kinetic energy that the molecules of a body or substance possess; the greater the movement of these, the higher the temperature of the body or substance. To make a temperature measurement it is necessary to use an intermediate device, for example, a glass thermometer where what is measured is the expansion of the mercury column, or a temperature sensor, where what is measured is the change in electrical voltage or resistance (Figure 1) [2].

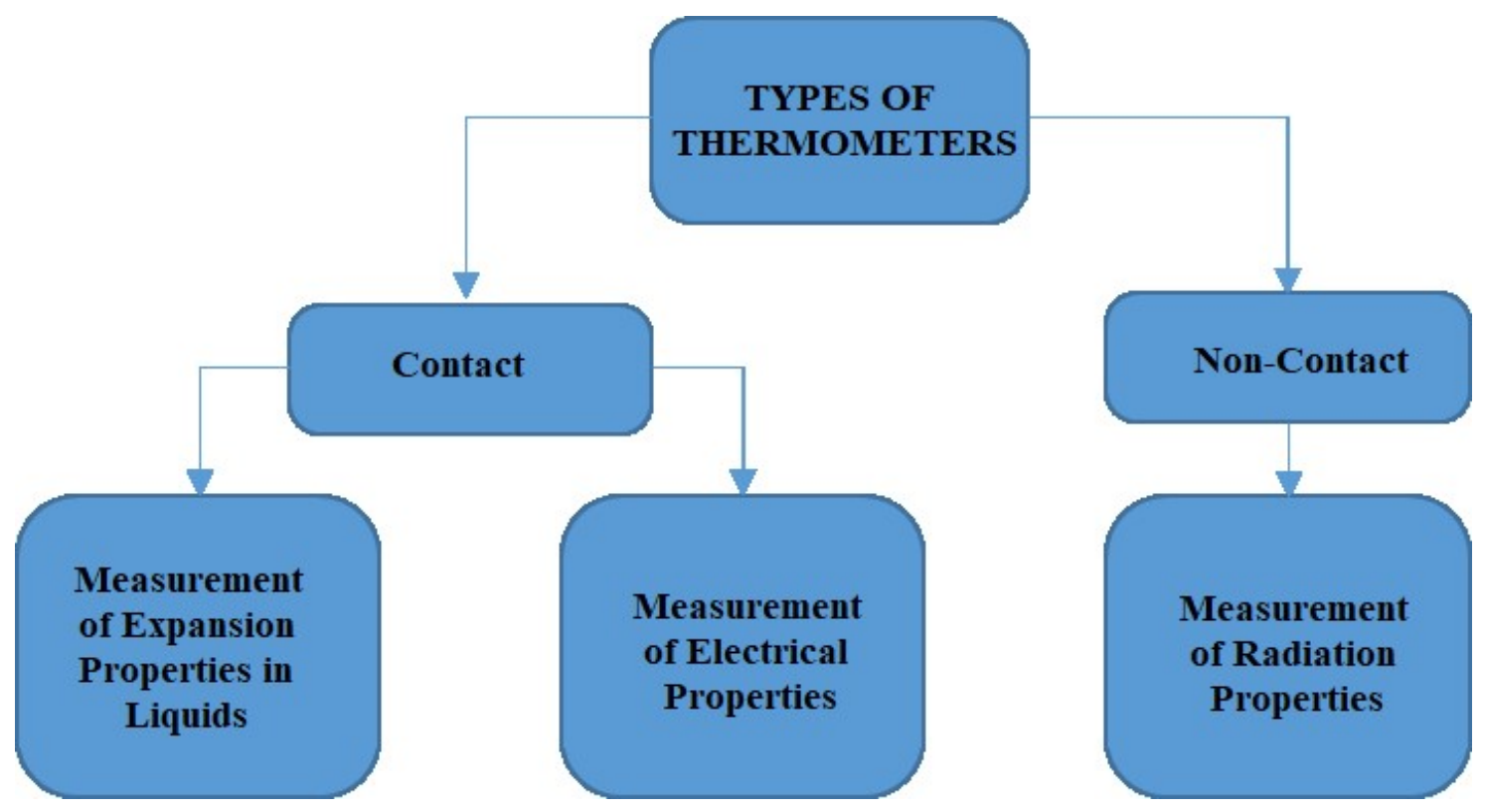

Figure 1: Types of thermometers [2] 
Since no thermometer remains constant permanently, because its characteristics change with time or with temperature cycles, it is necessary to characterize or calibrate the instrument. Calibration makes it possible to know the measuring characteristics of the thermometer, thus generating confidence in the measuring results obtained.

Applying Zeroth's law of thermodynamics to a calibration process by comparison in temperature, we can say that, if a calibrated thermometer is at the same temperature as a comparison medium and a thermometer under test is at the same temperature as a comparison medium, then the calibrated thermometer and the thermometer under test are at the same temperature.

In the previous case, the comparison medium must provide a constant temperature homogeneously and stably, because, without this, the required thermal equilibrium is not achieved. Additionally, not obtaining these characteristics will negatively influence the quality of the calibration results. A widely used means of comparison in the calibration of thermometers, especially platinum resistance ones, is the high-temperature oven [3], which must be configured to obtain control over its temperature variations. Due to the high temperatures that this equipment reaches, its manipulation generates a risk of accident for the user. Also, data collection, being a manual process, requires permanent intervention by the user.

With the implementation of this project, the aim is to develop a module in LabView ${ }^{\circledR}$ that allows, on the one hand, the parameterization of the operating conditions of the high-temperature furnace, specifically the "set point" temperature required by the user for the calibration process and, on the other hand, the automatic acquisition of data at an interval of 72 continuous hours without the need for user supervision.

\section{DESCRIPTION OF SYSTEM USED}

The high-temperature furnace used as a means of comparison in the calibration of Platinum Resistance Thermometers (PRT) is equipped with two independent control systems, one for temperature control and the other for overheating protection. The first one is the operation controller and it allows to control of the desired reference temperature value. It has several physical connections that, when improperly configured, can disable key functions of the furnace such as alarms or operation times, among others.

The protection controller is intended to set an upper-temperature limit, after which the power supply will be cut off to protect the system against the effects of a possible failure of the first controller. In use, this controller is set to a temperature of approximately $50^{\circ} \mathrm{C}$ higher than the operating controller setting, this on the recommendation of the manufacturer.

Although these controllers allow adequate stabilization of the reference temperature and provide safety in the operation of the furnace, they are insufficient for the work required in the laboratory, since continuous user intervention is necessary for parameterization and the uninterrupted collection of measurement data. Currently, there are drivers developed in LabView ${ }^{\circledR}$ designed for this purpose; however, it was determined that these are designed to be used in the Eurotherm $24 x x$ [4] family of controllers, since when implemented with the high-temperature furnace controller, which has a 2600 family controller installed, it does not recognize the LabView ${ }^{\circledR}$ driver, generating communication errors and therefore in the configuration and correct operation of the furnace.

On the other hand, it is important to mention that the calibration of a PRT-type thermometer requires an approximate time of 72 hours, during which time data should be taken from measurements made in short periods. 


\section{THEORETICAL BASES}

\subsection{Temperature and Related Basics}

The electrical resistance of many metals (e.g. copper, silver, aluminum, platinum) increases approximately linearly with temperature, a characteristic that makes them useful as temperature sensors. This characteristic is measured by applying a current (AC or DC) through the metal and measuring the electrical voltage produced with a resistive bridge or a suitable voltmeter. This physical principle is used in platinum resistance thermometers (PRT), which determine temperature by measuring the electrical resistance of a piece of pure platinum wire. The reading obtained is converted to temperature using a calibration equation.

The length and diameter of the platinum wire used in a thermometer are often chosen so that the resistance of the device, when it is around $0^{\circ} \mathrm{C}$, is $100 \Omega$. This type of sensor is called a PT100 and its resistance changes by approximately $0.4 \Omega$ per ${ }^{\circ} \mathrm{C}$, i.e., using a typical measurement current of $1 \mathrm{~mA}$, at about $0{ }^{\circ} \mathrm{C}$, a PT100 sensor would have a voltage drop of about $100 \mathrm{mV}$ across its terminals and this would change by approximately $0.4 \mathrm{mV}$ per ${ }^{\circ} \mathrm{C}$. To verify that the PRT maintains these conditions when making a measurement, it is necessary to use a calibration process for the instrument.

Calibration of a platinum resistance thermometer requires the use of a fixed-point cell as the primary standard, which must reach a certain temperature either by cooling or heating. [5]. In this case, an aluminum cell with a solidification temperature of $660.323^{\circ} \mathrm{C}$ is used. To reach this temperature, it is essential to use a high-temperature furnace with a special design in its construction, such as the one shown in Figure 2, which is capable of developing and maintaining the temperature required by the aluminum fixed-point cell. The heat transfer from the furnace to the cell is done by convection so that the metal deposited in the cell obtains the temperature and calibration can proceed. As a means of comparison, the furnace must provide a constant, homogeneous and stable temperature to the aluminum fixed-point cell, so that it can provide the required temperature for the calibration of the PRT.

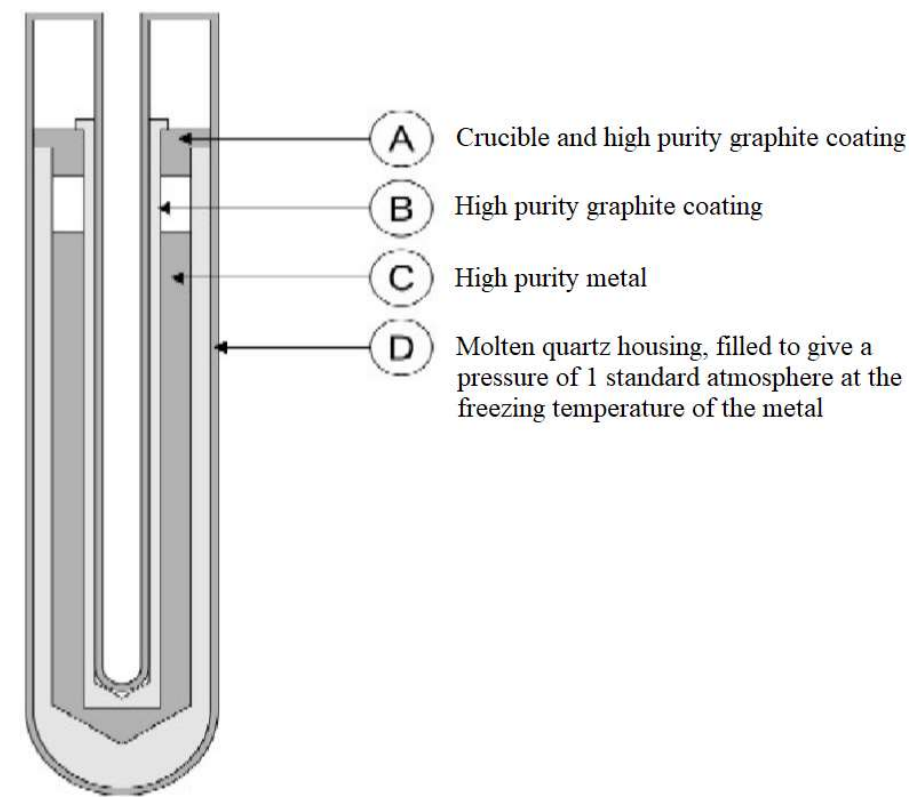

Figure 2: Sealed metal freeze point cell [6] 
It is essential to constantly monitor the temperature value in the oven to ensure that the calibration process is carried out correctly and that the results obtained are valid and consistent for subsequent analysis.

\subsection{Communication Interface and Protocol}

An interface is a physical port through which communication signals are transmitted and received between two or more systems. However, the interface alone is not capable of understanding the messages that generate these signals. To decipher the code, the message must have a structure and rules that define it. A data communication protocol defines these rules and the structure of the messages used by all devices in a network for data exchange. This protocol also defines the orderly exchange of messages, and error detection.

The oven has an RS422 interface which requires the use of an RS422 to RS232 converter cable for connection to a computer, as shown in Figure 3.

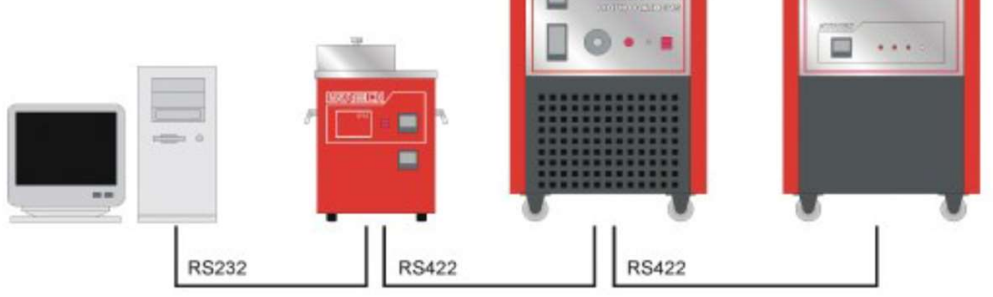

Figure 3: Oven Communication Interface [6]

This interface is also used for the communication of the PC with the main controller of the oven. Since only the oven will be connected to a PC for parameterization and data acquisition, with a distance of fewer than 15 meters [7], the RS232 standard is suitable for current communication needs.

Regarding the communication protocol, the controller allows different types, these are Modbus (or JBUS), EIBisynch, Profibus and Devicenet. Since in this work a master-slave scheme is configured, since communication always takes place in pairs (a device initiates a request and then waits for a response and the starting device (the master) is responsible for initiating each interaction), the Modbus protocol becomes the appropriate choice for communication between the oven and the PC [8]-[10].

The transmission mode used by Modbus is RTU. The configuration characteristics of this protocol are shown in Table 1.

Table 1: Modbus protocol description

\begin{tabular}{|l|l|}
\hline Feature & Description \\
\hline Baud Rate & 9600 (Default) \\
\hline Parity & None (Default) \\
\hline Address & $1-254$ \\
\hline Resolution & $\begin{array}{l}\text { Integer (16 bits) Default } \\
* \text { Full resolution floating-point data (32 bits) }\end{array}$ \\
\hline Transmission mode & RTU (Remote Terminal Unit) \\
\hline
\end{tabular}


One of the main limitations of Modbus is that only 16-bit integer data representations can be transmitted. To solve this, it is necessary to use a sub-protocol that uses the upper portion of the Modbus addressing space ( $8000 \mathrm{~h}$ and higher) allowing the full 32-bit resolution for floating-point data.

According to the controller communication manual [11], the RTU definition of the transmission mode for a single character consists of a start bit, eight data bits, a parity bit, and one or two stop bits. The parity can be set to None, Odd or Even. If the parity is set to NONE, no parity bits are transmitted. On the other hand [11], "a message consists of several characters sequenced so that the receiving device can understand it. This structure is known as the message format. Table 2 shows the sequence defined for the message format for the Modbus protocol.

Table 2: Message format in Modbus RTU

\begin{tabular}{|l|l|}
\hline Segment & Number of bytes \\
\hline Start of frame & 3 \\
\hline Address of the device & 1 \\
\hline Function code & 1 \\
\hline Data & $\mathrm{n}$ \\
\hline Cyclic redundancy check (CRC) & 2 \\
\hline End of Transmission (EOT) & 3 \\
\hline
\end{tabular}

"The start of the frame is a period of inactivity of at least 3.5 times the transmission time of a single character. For example, at 9600 baud a character with 1 start bit, 1 stop bit and 8 data bits will require a message format of $3.5 \mathrm{~ms}$.

The device address is a single byte for each device on the network. The function code is a single instruction byte that describes the action to be performed by the slave.

The data segment of the message will depend on the function code and the number of bytes in the segment will vary accordingly. Typically, the data segment will contain the parameter address and the number of parameters to read or write. The cyclic redundancy check is an error verification code and is 2 bytes long. The end of transmission or EOT is a period of inactivity equivalent to 3.5 times the transmission time of a single character".

\section{MATERIALS AND METHODS}

\subsection{Hardware}

The following elements are used in the instrumental part:

- High-temperature oven with a heating range from $125^{\circ} \mathrm{C}$ to $1090{ }^{\circ} \mathrm{C}$.

- Process controller.

- $\mathrm{RS} 232$ interface

- RS422 Interface

- Computer

The interface is used for the communication of the oven and the controller with the application.

\subsection{Software}

LabView is a graphic programming environment that allows the development of applications for data acquisition, 
measurement analysis, instrument control, among other functions [12]. This powerful software has a Modbus library, which is composed of a set of virtual instruments that provide communication from any standard Ethernet or serial port. The resulting files are called VI (Virtual Instrument) and sometimes they can be separated due to their size. In some cases, one VI may contain another, thus forming what is called a subVI.

Using LabView the application is developed, designed for a sequence of three frames. In the first frame, the data file is created in which the oven temperature values are to be stored. The data in the generated file is of the string type, allowing the user to visualize the information in an Excel spreadsheet for manipulation and analysis.

In the second frame, the Modbus address coding is programmed, where the value of the oven's "set point" temperature is written. The data type of this parameter is single, which is 32 bits, allowing 6 digits of precision. This aspect is key because if it is not correctly configured, it can produce an error in the communication between the application and the oven. Additionally, it is programmed the conversion of real type numerical data, corresponding to the temperature value of "set point" to be obtained, to a binary value, which is combined in an arrangement of one dimension to send and write the data in the direction of the controller of the oven, according to the addresses presented in Figure 4. Finally, the second frame contains the programming for the user to establish in the controls of the graphical interface the number of decimal digits required for reading according to their needs. Once the data is sent, the application waits $60 \mathrm{~ms}$ to read the Modbus register, clean it and continue with the cycle.

\begin{tabular}{|c|c|c|c|}
\hline & \multirow[t]{2}{*}{ Home list } & \multicolumn{2}{|c|}{2400 Version 3} \\
\hline & & Modbus & Bisynch \\
\hline & Process Variable & 1 & $\mathrm{PV}$ \\
\hline$\square P$ & $\%$ Output level & 3 & OP \\
\hline 5םPu & Valve position & & \\
\hline $5 P$ & $\begin{array}{l}\text { Target setpoint (if in Manual } \\
\text { mode) }\end{array}$ & 2 & SL \\
\hline$m-A$ & $\begin{array}{l}\text { Auto-man select } \\
0: \text { Auto } \\
\text { 1: Manual }\end{array}$ & 273 & $\mathrm{~mA}$ \\
\hline$A_{m} P 5$ & $\begin{array}{l}\text { Heater current (With PDSIO } \\
\text { mode 2) }\end{array}$ & 80 & LI \\
\hline$[\pi d$ & $\begin{array}{l}\text { Customer defined identification } \\
\text { number }\end{array}$ & 629 & ID \\
\hline P.5P & $\begin{array}{l}\text { Working set point. Read only: } \\
\text { use Target set point or } \\
\text { currently selected set point ( } 1 \\
\text { to } 16) \text { to change the value }\end{array}$ & 5 & $\mathrm{SP}$ \\
\hline
\end{tabular}

Figure 4: Modbus parameter addresses [11]

In the third frame, the application operation design pattern is created, as shown in Figure 5.

This pattern corresponds to a MASTER/SLAVE pattern. In the while cycle associated with the MASTER, readings are taken from addresses 1 and 5 of the controller. From these directions are obtained, according to Figure 4, the value of the temperature of "set point"(5) and the value of the current operating temperature of the oven (1). In the while cycle associated with the SLAVE, the writing of the variables on the front panel (individual numerical values, the data table + and the graph) is performed, the analysis of whether the value is stable is made, the number of decimal digits desired by the user for displaying is processed and the creation of a new file for the calibration process required by the laboratory (user) after the 
oven heating process is allowed. For this purpose, a subVI is used, which uses cluster type input and output data, that is, data from different formats are grouped (arrays, strings, integers, etc), which facilitates communication between the different VI's of the application.

Finally, the user can modify in the graphic interface the sampling period with which he requires the oven temperature data to be acquired during the calibration process.

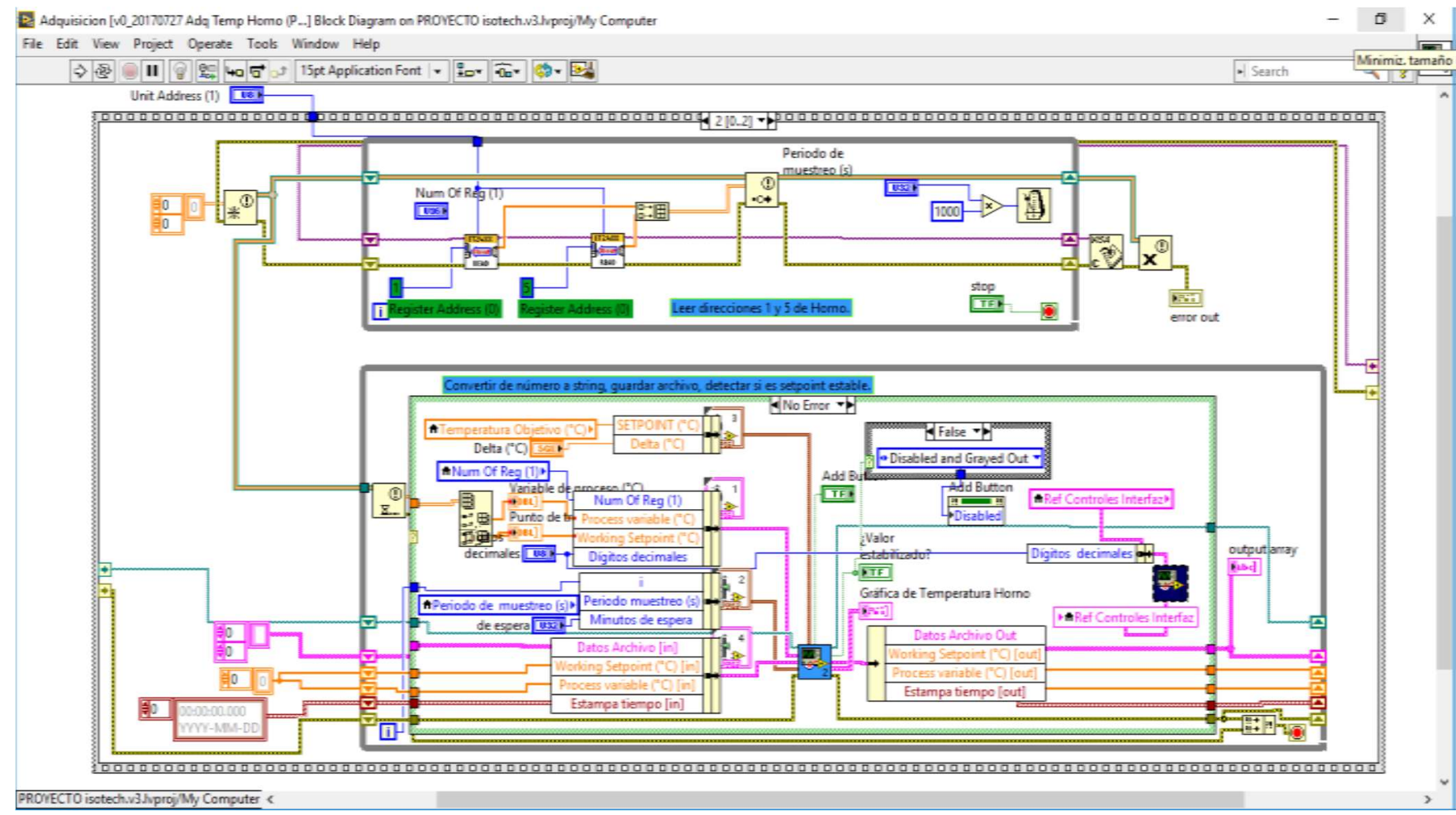

Figure 5: Application pattern design [13]

\section{RESULTS AND ANALYSIS}

After carrying out the tests, satisfactory results were obtained, since it was possible to evidence the correct communication between the devices through the Modbus protocol, which allowed, on the one hand, to properly parameterize the "set point" of the temperature and on the other hand to acquire uninterrupted data on the working temperature of the oven, as shown from Figure 6 to Figure 8. 


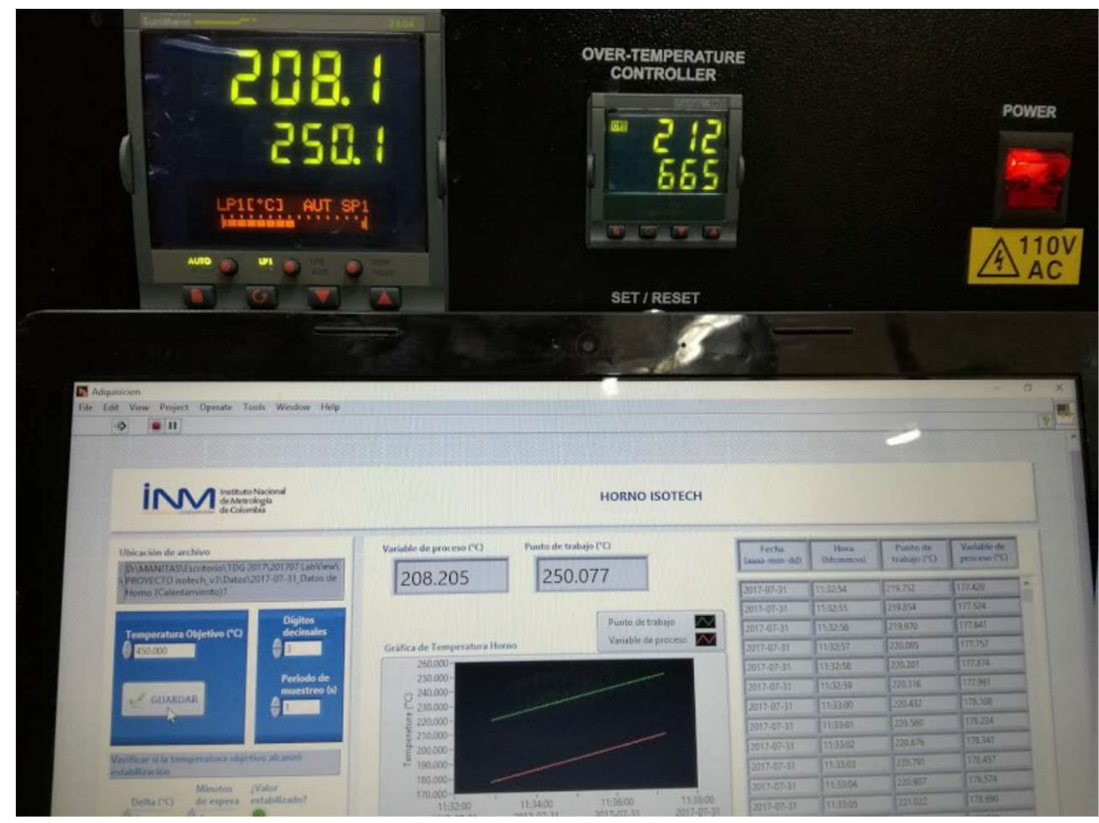

Figure 6: Evidence of operation and validation 1 [14]

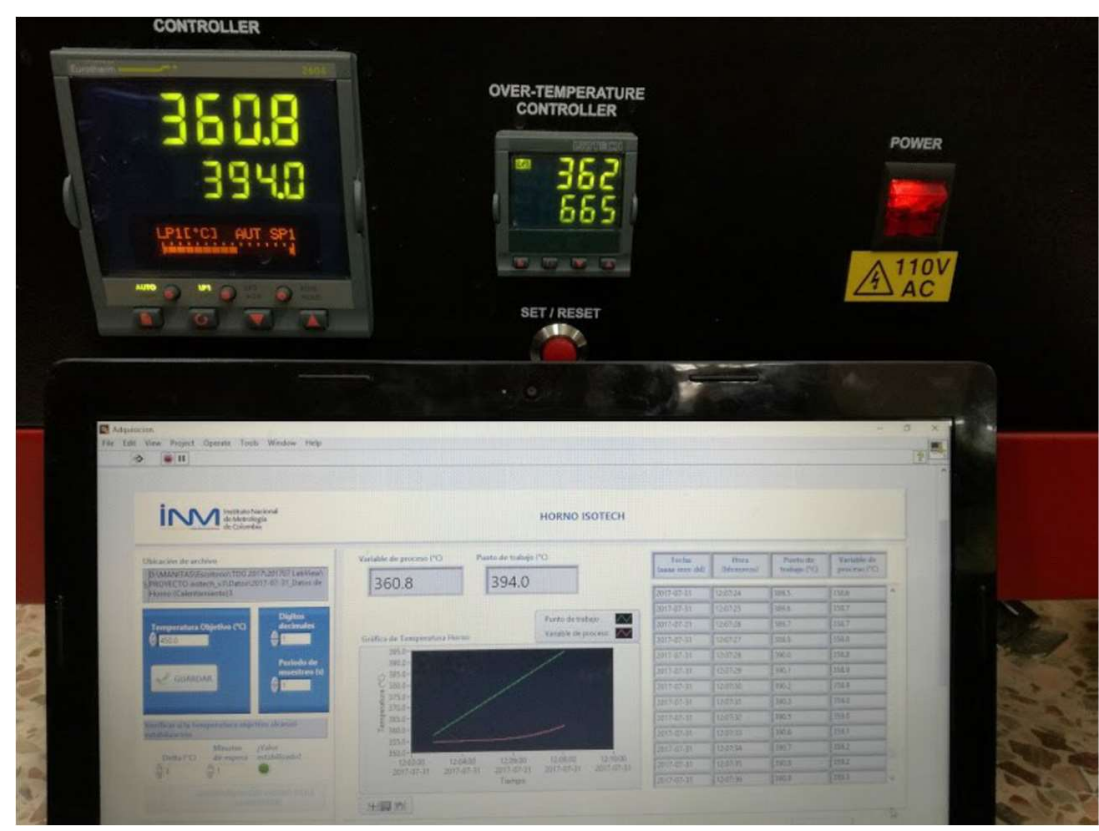

Figure 7: Evidence of operation and validation 2 [14] 


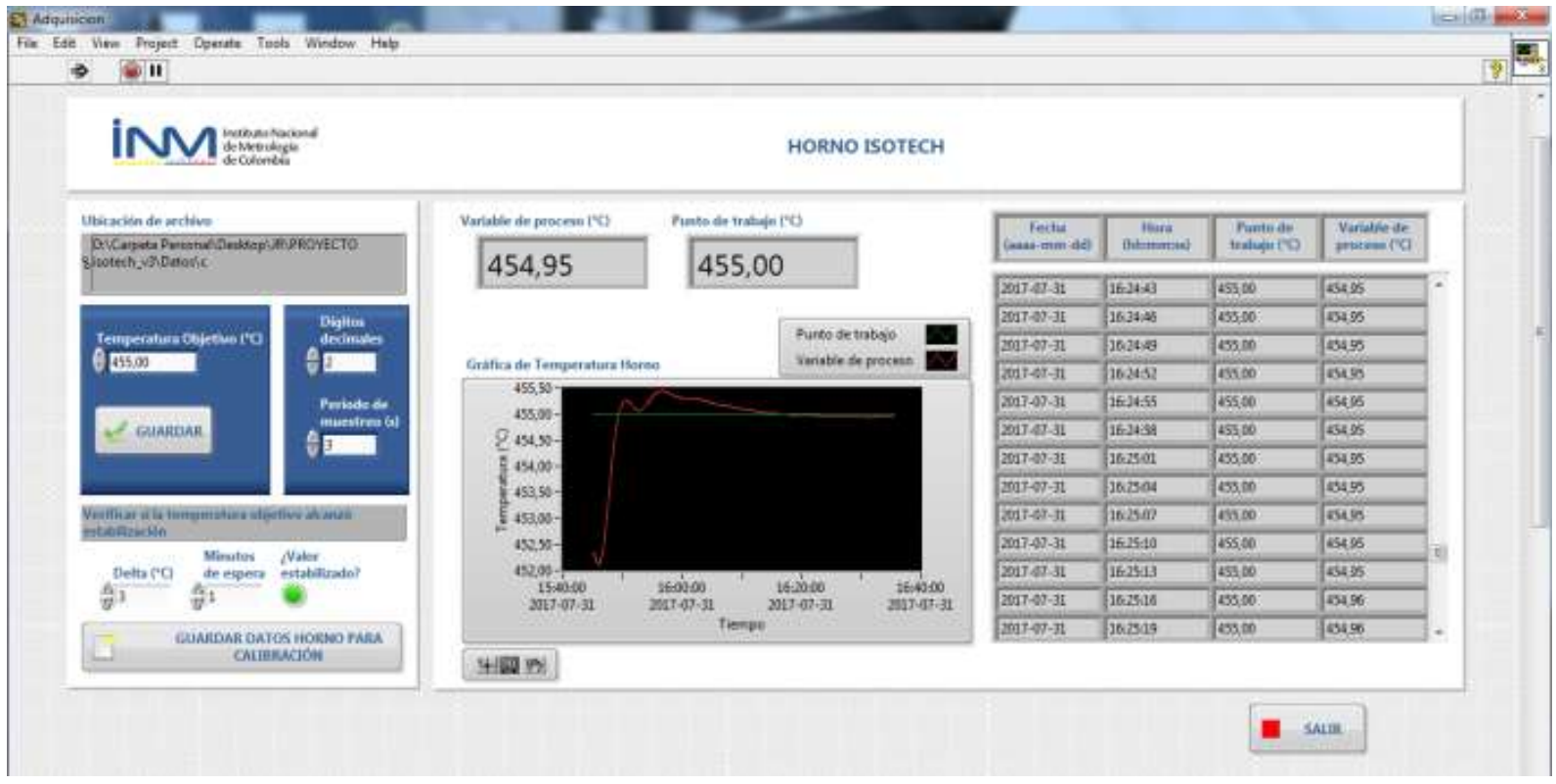

Figure 8: Evidence of operation and validation 3 [14]

The execution structure in LabView used was the sequence structure associated with the programming of the VI of communication and simultaneous presentation of the data in the user interface. The sequence-structure allows for execution code sections sequentially, that is, it allows to control the order of the execution and the data flow through "frames" (subdiagrams or tables). For example, the data reading subchart is only enabled after the user has set the target temperature.

In the file resulting from data acquisition, new rows of acquired data are added to those already written with each iteration of the application, constantly updating it. This makes it easier for the user to consult the file since he does not have to wait for the calibration process to be completed to access the information contained in the file.

\section{CONCLUSIONS}

In the configuration of the MODBUS protocol, complexity was found in several layers, therefore, instead of implementing a LabView ${ }^{\circledR}$ application that requires detailed knowledge of the protocol, such as the method of calculating the CRC (Error check data), tools were selected to apply the knowledge of the general structure of the protocol and a programming design pattern, thus optimizing the user interface of the final application.

The application becomes an element to carry out the metrological securing of the furnace with auxiliary equipment for the calibration of the aluminum fixed point.

\section{REFERENCES}

1. Fischer, J. \& Fellmuth, B. (2005). Temperature metrology. Reports on Progress in Physics. 68(5):1043-1094.

2. National Physical Laboratory. (2016). Good Practice Guide No. 125 - Introduction to Temperature Measurement. Retrieved from:https://www.npl.co.uk/special-pages/guides/gpg125_intro2tempmeasure

3. Eurotherm. (2007). 2216e Temperature controllers - Installation and Operation Handbook. Retrieved from: https:/www.eurotherm.com/download/2216e-installation-and-operation-guide-ha026639-iss-

$\underline{4 / \text { ?pdmdl }=26675 \& \text { refresh }=5 \mathrm{fbd} 935 \mathrm{a} 48 \mathrm{~b} 461606259546}$ 
4. Eurotherm. 2400 Series LabVIEW 8.0 Driver. Retrieved from: https://www.eurotherm.com/download/2400-series-labview-8-0driver/

5. Preston -Thomas, H. (1990). The International Temperature Scale of 1990 (ITS-90). Metrologia. 27:3-10.

6. Isotech. (2005). High Temperature Furnace Handbook Issue 8 - Model ITL- M-17702. Retrieved from: http://www.isotechna.com/v/vspfiles/product_manuals/Isotech/ITL-M-17702.pdf

7. Forero, N. G. (2012). Normas de Comunicación en Serie: RS-232, RS-422 y RS-485. Ingenio Libre. 11(1):86-94.

8. Salazar, R. (2006). Modbus RTU. Implementación del protocolo en microcontrolador. Trabajo de Grado. Universidad Industrial de Santander. Facultad de Ingenierías Fisicomecánicas. Bucaramanga. 2006

9. Stack overflow. (2015). Modbus4J Modbus RTU master. Retrieved from: http://stackoverflow.com/questions/32432548/modbus4j-modbus-rtu-master

10. Fauzi, E., Fajri, M. \& Iriani, Y. (2019). Architecture of Product Digital Scales Modbus Protocol-Based. International Journal of Advanced Science and Technology. 28(6):62-67.

11. Eurotherm. (2011). Series 2000 Communication Handbook. Retrieved from: http://controls.diamond.ac.uk/downloads/support/eurotherm2k/1-22/documentation/HA026230_3_2000\%20comms.pdf

12. Patel, B. N., Patel, J. B. \& Zadfiya, R. L. (2014). Development of a Scada System for Temperature Control Using Lab View. International Journal of Electronics, Communication \& Instrumentation Engineering Research and Development (IJECIERD). 4(2):113-118.

13. Montaño, Andrés. (2017). Diseño de patrón de la aplicación. Instituto Nacional de Metrología, Bogotá, 2017.

14. Montaño, Andrés. (2017). Evidencia de funcionamiento y validación. Instituto Nacional de Metrología Bogotá, 2017. 